\title{
Reproductive Factors and Breast Cancer Risk
}

\author{
Juveria Tarannum ${ }^{1 *}$, Pittala Manaswini' ${ }^{1}$, Chekoorthi Deekshitha ${ }^{1}$, Raj Kumar Gaju ${ }^{1}$, Anchuri Shyam Sunder ${ }^{1}$ \\ ${ }^{1}$ Department of Pharmacy Practice, Balaji Institute of Pharmaceutical Sciences, Telangana, India
}

Corresponding Author: Juveria Tarannum, Pharm.D, Department of Pharmacy Practice, Balaji Institute of Pharmaceutical Sciences, Telangana, India. Tel: +91-8333047446, Email: juveria5496@gmail.com

Received November 23, 2018; Accepted May 2, 2019 ; Online Published June 15, 2019

\begin{abstract}
A neoplasm, also referred to as a tumor, is an abnormal mass of tissue, the growth of which exceeds and is uncoordinated with that of normal tissue and remains in the same excessive manner even after removal of the stimuli which evoked the changes. Tumors may be either benign or malignant. Benign tumors remain localized and are amenable to surgical removal, whereas malignant tumors, i.e. cancerous ones, adhere to any part in an obstinate manner, often invade surrounding tissue, and metastasize to distinct sites. Breast cancer occupies second place as the most occurring cancer in terms of incidence. Breast cancer is a heterogenous cancer, mostly linked with reproductive and hormonal factors in its occurrence. Nulliparous, younger age at menarche, early age pregnancy, older age at first live birth, late menopause, prolonged interval between menarche and first pregnancy, repeated abortions, first pregnancy after 35 years, and no/less breastfeeding are major risk factors for breast malignancies. Recent studies have shown that the implications of reproductive and hormonal factors in patients with breast cancer are associated mostly with BRCA1 (breast cancer gene 1) and BRCA2 (breast cancer gene 2) gene mutations.

Keywords: Reproduction, Breast Neoplasms, Menarche, Menopause, Neoplasm Metastasis

Citation: Tarannum J, Manaswini P, Deekshitha C, Gaju RK, Sunder AS. Reproductive factors and breast cancer risk. Int J Med Rev. 2019;6(2):4044. doi:10.29252/IJMR-060203.
\end{abstract}

\section{Introduction}

A risk factor is defined as anything that increases the probability of developing diseases in a person. ${ }^{1,2}$ Breast cancer is a clinically heterogeneous and complex malignancy which occupies first place in women in terms of incidence around the world. Hence, it constitutes a major public health concern in both developed and developing countries as a female cancer risk. ${ }^{3}$ Over the past decade, it has been evident that breast cancer represents as a heterogeneous disease, for which different subtypes are distinguished based on the combination of tumor grade and presence of hormone receptors, i.e. estrogen (ER), progesterone (PR), and human epidermal growth factor receptor-2 (HER2). Breast cancer subtypes, like luminal A-like (ER+/PR+,HER2-ve, grade 1 or 2), luminal B-like (ER+/PR+, HER2-ve, grade 3), luminal HER2-like (ER+/PR+ and HER2+), HER2-like (ER-ve, PRve, HER2-positive), and triple negative breast cancer (TNBC) (ER-ve, PR-ve, HER2-ve), present with different risk factors associated with them. ${ }^{4}$

Analyses from the Breast Cancer Association Consortium (BCAC) show that nulliparity and first full-term pregnancy (FFTP) at a later age increase the risk of ER+ve breast cancer. Reproductive and hormonal factors frequently contribute to the development of breast cancer. Nulliparous, younger age at menarche, early age at pregnancy, older age at first live birth, late menopause, prolonged interval between menarche and first pregnancy, repeated abortions, first pregnancy after 35 years, and no/less breastfeeding are major risk factors for breast malignancies. ${ }^{5-8}$ Recent studies have shown that the implications of reproductive and hormonal factors in patients with breast cancer are associated with BRCA1 and BRCA2 gene mutations. ${ }^{9}$

Hormonal and reproductive factors are associated with breast cancer through prolonged exposure to ER. This explains why these factors are most commonly associated with hormonal histological abnormalities. There are two theories regarding this risk associated with breast malignancies. The first mechanism is induced transcription mediated by ER receptors, which determines cell proliferation; the second is stimulation of carcinogenesis due to metabolic activation and direct DNA binding. These two mechanisms, either individually or combined, mutually reinforce breast malignancies. ${ }^{10}$

\section{Tumorigenesis}

Breast tumors usually start from ductal hyper proliferation and then develop as benign tumors or even as metastatic carcinomas after constant stimulation by various carcinogenic factors. Tumor microenvironments such as stromal influences or macrophages are important in breast cancer initiation and progression. Macrophages generate a mutagenic inflammatory microenvironment, which promotes angiogenesis and

Copyright (C) 2019 The Author(s). This is an open-access article distributed under the terms of the Creative Commons Attribution License (http:// creativecommons.org/licenses/by/4.0), which permits unrestricted use, distribution, and reproduction in any medium, provided the original work is properly cited. 
enables cancer cells to escape the immune rejection. Different DNA methylation patterns are observed in normal and tumor-associated microenvironments, indicating epigenetic modifications in the tumor microenvironment which promote carcinogenesis. Recently, it was determined that cancer stem cells (CSCs) are associated with tumor initiation, escape, and recurrence. These CSCs develop from progenitor cells in normal tissues, which are resistant to chemotherapy and radiotherapy. ${ }^{11}$

ER levels play an important role in reproductive events. Increases in ER levels lead to menarche, decreasing levels precipitate menopause, and ER augmented with PR promotes cell division, which increases the chances of mutated cell growth. ${ }^{12}$ Histology revealed that terminal duct lobular units (TDLUs) are the origin of breast cancer in women, and the ER and PR receptors are expressed only in luminal epithelial cells of ducts, as these cells are the initial sites of malignant transformation. ${ }^{9}$ Malignant cells give rise to ductal carcinoma in situ (DCIS), the most common histological variant of the non-invasive stage of breast cancer. They may also penetrate basal cells and extend to other parts of the body, resulting in invasive ductal carcinoma (IDC), which accounts for about $80 \%$ of all cases of invasive breast cancer. The endogenous levels of steroid hormones fall after menopause because of the cessation of ovarian activity. At this point, adipose tissue acts as a main source of ER, and high levels of estrone (E1) are associated with a higher risk of postmenopausal breast malignancies. High levels of estradiol and testosterone are also associated with increased risk of breast cancer (Figure 1). ${ }^{9}$

\section{Subtypes}

Luminal A and Luminal B Breast Cancer

Luminal A and Luminal B breast cancers are characterized by high expression of ER and gene expression similar to normal cells lining the breast ducts and glands. HER2 is expressed mostly by Luminal B breast cancer. Luminal breast cancers are generally low grade and grow slowly. Luminal A cancer has a better prognosis than Luminal B cancer.'

\section{HER2 Breast Cancer}

In HER2 breast cancer, the over-expression of HER2 is seen.

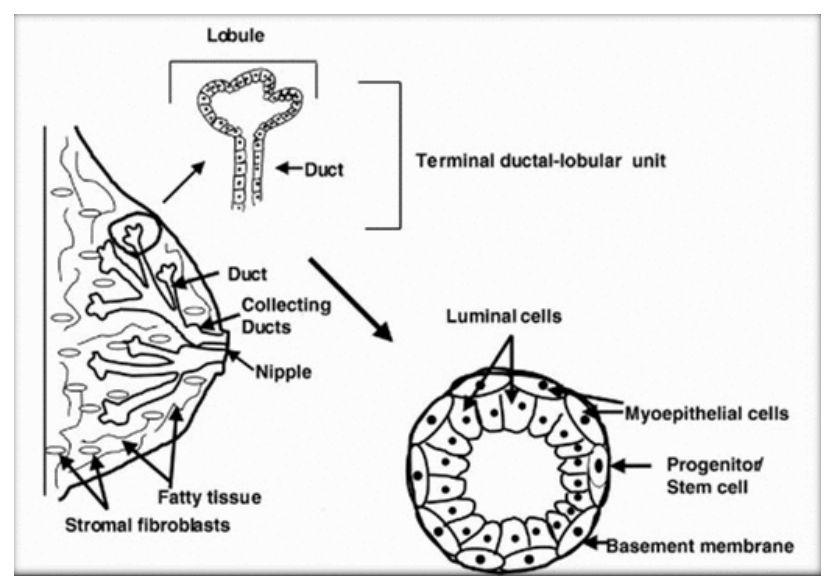

Figure 1. Structure of the mammary gland and terminal ductal-lobular unit (TDLU). ${ }^{9}$
HER2 breast cancer is generally a high-grade tumor, and these tend to grow rapidly and are associated with poor prognoses. ${ }^{9}$

\section{Basal-Like Breast Cancer}

Basal-like breast cancer is characterized by a lack of ER/PR expression and has the pattern of gene expression characteristic of breast basal epithelial cells, such as cytokeratin 5/6. These are high-grade tumors which grow rapidly and are associated with poor prognoses. ${ }^{9}$

\section{Reproductive Risk Factors \\ Menstruation}

Menarche at a young age is associated with an increased risk of positive hormone receptors in breast carcinoma ( $\mathrm{HR}+)$; the risk is highest for people in whom menarche occurs before 12 years of age. $^{2}$ It seems that menarche at a young age is associated with histological tumor with the over-expression of human epidermal receptor 2 protein (HER2+). Furthermore, different studies suggest that early menarche is also associated with the risk of triple negative breast cancer (TNBC). Each year delay in the occurrence of menarche decreases the risk of breast cancer by $7 \%$ for both premenopausal and postmenopausal women. ${ }^{10}$ Many studies have stated that women aged between 14-15 years at menarche have a 54\% reduction in breast cancer risk compared with women who had menarche at age $<12$ years, as it causes the prolonged exposure of breast epithelia to ERs, and such women have increased levels of estradiol and high levels of ER throughout their reproductive lives. Higher levels of estradiol along with low levels of sex hormone-binding globulin are seen in women with early menarche, allowing free estradiol to have more bioavailability to the breast. ${ }^{7,13}$ Many studies have stated that women with early menarche have higher ER levels than women with late menarche for several years after menarche, which leads to prolonged exposure of the breast epithelia to high ER levels produced by regular ovulatory cycles. ${ }^{14}$ Early age at menarche ( $<13$ years old) increases the risk of luminal tumor compared with women who had menarche at 15 years of age. ${ }^{15}$ Yang's meta-analysis of 12 populationbased studies explained that 1.16 times higher ER+ tumor risk is seen in women who experience an early menarche $(<12$ years). Late menopause is associated with the risk of ductal types and lobular types of breast malignancies as well as HR+ histological type breast cancers in well-differentiated (G1) forms. ${ }^{3}$ Menarche and menopause bring marked changes within the breast and are related to hormonal fluctuations and cellular proliferation. ${ }^{16}$

\section{Age at Menopause}

Before menopause, serum ovarian hormones circulate freely in the blood and have bioavailability to the breast, resulting in the proliferation of breast epithelial cells. At the time of menopause, the exposure of mammary epithelial cells to sex hormones is decreased, resulting in decreased mammary epithelial cell proliferation. Having an older age at menopause maximizes the number of ovulatory cycles for women and therefore increases the risk of breast malignancy. The risk in women who had natural menopause at age 55 or more is twice 
that of women who experience menopause at age 44. Women who had a surgical hysterectomy or oophorectomy before the age of 35 years have a much lower breast cancer risk. ${ }^{2,7}$ Young age at menarche and old age at menopause are highly linked with breast cancer. ${ }^{15,17,18}$

\section{Parity}

Several studies have suggested a relationship between exposure to pregnancy hormones and breast malignancies, especially multiparous status and breast cancer risk are in conflict. ${ }^{19}$ Mostly, the events during a woman's first pregnancy, when breast tissue is less differentiated, are susceptible to mutagenic changes. Most studies show an inverse association between the number of pregnancies and risk of $\mathrm{HR}+$ breast cancer types. In other cases where there is a positive family history of breast malignancies, parity is associated directly with breast malignancies. This is supported by Hajiebrahimi et al, in a study in 2015 on women with singlet pregnancies who gave birth in 1973-2010 compared to their siblings. They concluded that the risk of breast cancer increased with parity between the two groups. ${ }^{9,10,20,21}$ Parity has a protective role in the occurrence of breast cancer in postmenopausal women, but this is inversely related with premenopausal women. ${ }^{22}$ This protective effect of parity is seen only in HR+ types; TNBC types are not influenced by parity status. ${ }^{10}$ Pregnancy causes accelerated differentiation of breast tissue and rapid proliferation of mammary epithelial cells. Breast cancers are related to the rate of proliferation of mammary epithelial cells and inversely related to the degree of differentiation, which decreases with subsequent pregnancies. Multiparous status has a protective effect against ductal types and ductolobular subtypes but not for lobular forms of malignancies. In univariate analysis, it was found that multiparous state decreases the risk of breast malignancies up to $74.2 \%{ }^{3}$ Higher parity is associated with extensive breast cancer mortality among premenopausal women. A large UK cohort study found that approximately a $10 \%$ reduction in risk for each additional birth for the most common histologic types of breast malignancies is seen. Each birth reduces the risk of $\mathrm{ER}+\mathrm{PR}+$ cancer by $11 \% .{ }^{23}$ The risk of breast cancer is 5 times higher (i.e. 4.5-9.4 times) in nulliparous women than parous women. ${ }^{14}$

Prolonged Interval Between Menarche and First Pregnancy Women who have their FFTP at $\geq 30$ years of age have a $50 \%$ increased risk of breast cancer compared with those women who have their FFTP at $<20$ years of age. ${ }^{6}$ This protective effect is validated for HR+ type, and the risk of TNBC remains unchanged. In a systematic review in 2014, Namiranian et al showed that a first pregnancy after 30 years of age and nulliparous women are strongly associated with the risk of breast cancer. ${ }^{24}$ The prolonged interval between menarche and first pregnancy is associated with $\mathrm{HR}+$ breast cancer, and a short interval between these events is related with TNBC. ${ }^{10}$ Mesharam et al explained that experiencing menopause at an age of $\geq 50$ years compared with women who had menopause before 45 years of age is associated with a 7.9-times greater risk of developing breast malignancies. ${ }^{14}$
Age at First Pregnancy

Pregnancy before 30 years of age has dual effects on the breast. First, patients experience a transient increase in breast cancer risk, followed by a progressive reduction in breast cancer risk after pregnancy. A late pregnancy (after 38 years of age) increases both short-term and long-term risks for lobular breast cancer. ${ }^{25}$ Advanced age at first birth increases risk up to $4 \%$ per year for breast cancer in premenopausal women and up to $2 \%$ per year for women in menopause. Women who have a late first pregnancy also have a higher risk of developing lobular breast carcinoma and/or HR+ breast cancer, and HER2 overexpression is seen in patients. Women who become pregnant by assisted reproduction techniques have a higher risk of breast malignancies compared with nulliparous women, if they have their first pregnancy at later than 35 years of age. ${ }^{10}$ Pregnancy at a younger age ( $\geq 20$ years) is associated with normal ER profiles, which reduces the presence of undifferentiated/vulnerable breast cells, which differentiates the terminal end lobules, reduces the pool of ER receptor positive cells, and acts as a protective factor against $\mathrm{ER}+$ breast cancer. ${ }^{26}$ The risk for breast malignancy is greater for women who have their first child after the age of 25 years compared with women who have their first child at or before the age of 20 years. ${ }^{14,19}$ A late age at FFTP is related to high ER levels, which transform the stem cells into tumor cells and, upon long exposure, into malignant cells. In the same manner, pregnancy projects a protective effect by differentiating the stem cells and making them resistant to carcinogenic effects. ${ }^{7,22}$ Tumors diagnosed during the first 2 years after pregnancy are highly aggressive with a higher stage of axillary lymph node involvement with high mitotic count and no expression of ER or PR cells. Women who experience FFTP at an older age are less likely to be diagnosed with TNBC; an increase in age at FFTP increases risk. ${ }^{4,27}$ Eighteen studies on age at FFTP showed that each additional year led to an increased risk of $1.05 \%$ for early breast cancer and $1.03 \%$ for late breast cancer. ${ }^{28}$ The earlier the FFTP occurs, the earlier the breast cells undergo differentiation and proliferation, which occurs between 10-20 years of age and facilitates promotion of the carcinogenic process. ${ }^{28}$

\section{Breastfeeding}

Breastfeeding acts as a protective factor against breast cancer through a hormonal mechanism, where the lactation stimulates the breast tissue differentiation and changes the ER levels from breast tissue. Moreover, prolactin induces lactation, which helps the excretion of carcinogenic agents from mammary ducts and probably reduces women's lifetime menstrual cycles. ${ }^{2,710,15,29}$ The inverse association between breastfeeding and risk of breast malignancies is explained by a biological mechanism, i.e. a reduction in ER levels. With an increase in the production of prolactin, women are less exposed to ER. ${ }^{30}$ Other studies have stated that the blood ER levels of nursing women gradually increase after the last birth and continue to increase for several years until they reach the nulliparous state. ${ }^{3}$ A study in 2002 analyzed the role of reproductive factors in breast cancer occurrence. It was agreed that the protective effect of breastfeeding is directly 
proportional to the duration of breastfeeding a mother has. Another study in 2016 reported that multiparous women who breastfed up to 12 months were at a lower risk for breast cancer of the Luminal B subtype, but those who breastfeed for more than 12 months have a higher risk of both luminal A and luminal B subtypes. ${ }^{10}$ Not breastfeeding or breastfeeding for a shorter duration is associated with the risk of breast cancer; mothers who do not breastfeed in their lifetime are at a higher risk for developing breast malignancy. The total duration of breastfeeding also plays a role in breast malignancy; as the total duration of breastfeeding increases, the risk of breast cancer decreases. ${ }^{14}$ Seven studies related to breastfeeding have explained that it reduces both ER+PR+ and ER-PR- cancer. ${ }^{23}$ A 2013 study by Li et al indicated that a reduction of up to $50 \%$ in the risk of TNBC-type breast cancer can be seen in women aged $(<45)$ who breastfed for 12 months compared with women who never breastfed. ${ }^{31}$

\section{Preterm Birth}

In their 1999 study, Melbye et al first demonstrated that the risk of breast cancer is $72 \%$ higher in women who have had at least one preterm birth (less than 31 weeks). ${ }^{32}$ This is explained by the fact that breast cells proliferate, especially in the first and second trimesters, while in the $3^{\text {rd }}$ trimester, the breast is in the process of maturation. With premature births, i.e. delivery at the end of the second trimester, the mammary gland remains in the proliferative state, which makes it susceptible to carcinogenic factors. Recent studies, including Innes et al, have explained that the risk of breast cancer is almost double in mothers who have had preterm births compared with mothers with full-term births, ${ }^{33}$ Hajiebrahimi et al also explained that preterm births are associated with a higher risk for mothers of developing breast cancer. ${ }^{10}$

\section{Conclusions}

Women who have had a cessation of menstruation have more adipose tissue in the breast which is associated with high levels of estrone (E1), which may result in postmenopausal malignancy. Prolonged exposure of breast epithelia to ERs is seen in early menarche, which is associated with increased levels of estradiol. Moreover, high levels of ER throughout one's reproductive life and low levels of the sex hormonebinding globulin are seen in women with early menarche, allowing the free estradiol to have more bioavailability to the breast, resulting in the risk for breast malignancy. Serum ovarian hormones, which circulate freely in the blood and have bioavailability to the breast, result in the proliferation of breast epithelial cells. At menopause, the exposure of mammary epithelial cells to sex hormones decreases, resulting in decreased mammary epithelial cell proliferation. Hence, late menopause maximizes the number of ovulatory cycles for women, and thus increases the risk of breast malignancy. Pregnancy results in the accelerated differentiation of breast tissue and the rapid proliferation of mammary epithelial cells. Breast cancers are related to the rate of proliferation of mammary epithelial cells, which decreases with subsequent pregnancies; hence, this proves that multiparous status has a protective effect against breast malignancy. Pregnancy has a protective effect by differentiating the stem cells and making them resistant to carcinogenic effects. Having a first pregnancy at a late age is associated with high ER levels which transform stem cells into tumor cells and, on long exposure, into malignant cells. Breastfeeding results from the increased production of prolactin which induces lactation which helps in the excretion of carcinogenic agents from mammary ducts. Breastfeeding also results in less exposure of the breast to ER, stimulating breast tissue differentiation, changing ER levels from breast tissue, and reducing the risk of breast malignancy.

\section{Authors' Contributions}

JT, PM, and CD contrived the idea in the title as well as its prevalence and importance in the incidence of cancer. JT performed the literature review search and completed the writing of the whole manuscript. ASS and RKG undertook the responsibility of organizing, supervising, and critically reviewing the article in the course of its generation.

\section{Conflict of Interest Disclosures}

The authors declare they have no conflicts of interest.

\section{References}

1. Balekouzou A, Yin P, Pmatika CM, et al. Reproductive risk factors associated with breast cancer in women in Bangui -a case-control study. BMC Womens Health. 2017;17:14.

2. Aich RK, Mondal NK, Chhatui B, et al. Relevance of risk factors of breast cancer in women: An Eastern Indian scenario. J Cancer Res Ther. 2016;12(1):302-308. doi:10.4103/0973-1482.160929.

3. Laamiri FZ, Hasswane N, Kerbach A, et al. Risk factors associated with a breast cancer in a population of Moroccan women whose age is less than 40 years: a case control study. Pan Afr Med J. 2016;24:19. doi:10.11604/pamj.2016.24.19.8784.

4. Brouckaert O, Rudolph A, Laenen A, et al. Reproductive profiles and risk of breast cancer subtypes: a multi-center case-only study. Breast Cancer Res. 2017;19(1):119. doi:10.1186/s13058-0170909-3.

5. Ritte R, Tikk K, Lukanova A, et al. Reproductive factors and risk of hormone receptor positive and negative breast cancer: a cohort study. BMC Cancer. 2013;13:584. doi:10.1186/1471-2407-13584 .

6. Palachandra A, Ishawaraprasad GD, Sreelatha CY, Sumana M. Risk factors associated with carcinoma breast: a case control study. Int Surg J. 2017;4(9);3136-3140. doi:10.18203/2349-2902. isj20173902.

7. Ahmad Kamruddin S. Breast cancer risk factors among MexicanAmerican women [dissertation]. The University of Texas School of Public Health; 2013.

8. Gao YT, Shu XO, Dai Q, et al. Association of menstrual and reproductive factors with breast cancer risk: results from the Shanghai Breast Cancer Study. Int J Cancer. 2000;87(2):295300. doi:10.1002/1097-0215(20000715)87:2<295::aidijc23>3.0.co;2-7.

9. Hajiebrahimi M. Reproductive factors with respect to breast cancer risk and breasts cancer survival. Stockholm: Karolinska Institutet; 2014.

10. Goidescu IG, Eniu DT, Caracostea G, Cruciat G, Stamatian F. Reproductive factors and breast cancer risk. Obstet Ginecol. 2016;LXIV(3):129-135.

11. Sun YS, Zhao Z, Yang ZN, et al. Risk factors and preventions of breast cancer. Int J Biol Sci. 2017;13(11):1387-1397. doi:10.7150/ ijbs.21635.

12. Phillips LS, Millikan RC, Schroeder JC, Barnholtz-Sloan JS, Levine BJ. Reproductive and hormonal risk factors for ductal 
carcinoma in situ of the breast. Cancer Epidemiol Biomarkers Prev. 2009;18(5):1507-1514. doi:10.1158/1055-9965.epi-08-0967.

13. Khalis M, Charbotel B, Chajes V, et al. Menstrual and reproductive factors and risk of breast cancer: A case-control study in the Fez region, Morocco. PLoS One. 2018;13(1):e0191333. doi:10.1371/ journal.pone.0191333.

14. Mesharam II, Hiwarkar PA, Kulkarni PN. Reproductive risk factors for breast cancer: A case control study. Online J Health Allied Sci. 2009;8(3):5.

15. Li H, Sun X, Miller E, et al. BMI, reproductive factors, and breast cancer molecular subtypes: A case-control study and meta-analysis. J Epidemiol. 2017;27(4):143-151. doi:10.1016/j. je.2016.05.002.

16. Oh $\mathrm{H}$, Bodelon $\mathrm{C}$, Palakal $\mathrm{M}$, et al. Ages at menarche- and menopause-related genetic variants in relation to terminal duct lobular unit involution in normal breast tissue. Breast Cancer Res Treat. 2016;158(2):341-350. doi:10.1007/s10549-016-3859-z.

17. Menarche, menopause, and breast cancer risk: individual participant meta-analysis, including 118964 women with breast cancer from 117 epidemiological studies. Lancet Oncol. 2012;13(11):1141-1151. doi:10.1016/s1470-2045(12)70425-4.

18. Mogren I, Stenlund $H$, Hogberg $U$. Long-term impact of reproductive factors on the risk of cervical, endometrial, ovarian and breast cancer. Acta Oncol. 2001;40(7):849-854. doi:10.1080/02841860152703481.

19. Steiner E, Klubert D, Knutson D. Assessing breast cancer risk in women. Am Fam Physician. 2008;78(12):1361-1366.

20. Warren Andersen S, Trentham-Dietz A, Gangnon RE, et al. The associations between a polygenic score, reproductive and menstrual risk factors and breast cancer risk. Breast Cancer Res Treat. 2013;140(2):427-434. doi:10.1007/s10549-013-2646-3.

21. Clavel-Chapelon F, Launoy G, Auquier A, et al. Reproductive factors and breast cancer risk. Effect of age at diagnosis. Ann Epidemiol. 1995:5(4):315-320. doi:10.1016/1047-2797(95)00099-S.

22. Tamakoshi K, Yatsuya H, Wakai K, et al. Impact of menstrual and reproductive factors on breast cancer risk in Japan: results of the JACC study. Cancer Sci. 2005;96(1):57-62. doi:10.1111/j.13497006.2005.00010.x.

23. Ma H, Bernstein L, Pike MC, Ursin G. Reproductive factors and breast cancer risk according to joint estrogen and progesterone receptor status: a meta-analysis of epidemiological studies. Breast Cancer Res. 2006;8(4):R43. doi:10.1186/bcr1525.

24. Namiranian N, Moradi-Lakeh M, Razavi-Ratki SK, Doayie M, Nojomi M. Risk factors of breast cancer in the Eastern Mediterranean Region: a systematic review and meta-analysis. Asian Pac J Cancer Prev. 2014;15(21):9535-9541. doi:10.7314/ apjcp.2014.15.21.9535.

25. Ewertz M, Duffy SW. Risk of breast cancer in relation to reproductive factors in Denmark. Br J Cancer. 1988;58(1):99-104. doi:10.1038/bjc.1988.172.

26. Anderson WF, Pfeiffer RM, Wohlfahrt J, Ejlertsen B, Jensen MB, Kroman N. Associations of parity-related reproductive histories with ER+/- and HER2+/- receptor-specific breast cancer aetiology. Int J Epidemiol. 2017;46(1):86-95. doi:10.1093/ije/dyw286.

27. Costantino NS, Cox TM, Shriver CD, Ellsworth RE. Lifestyle and Reproductive Factors Associated with Breast Cancer Risk in Young Women. EC Gynaecology. 2017;4(2):36-45.

28. Clavel-Chapelon F. Differential effects of reproductive factors on the risk of pre- and postmenopausal breast cancer. Results from a large cohort of French women. Br J Cancer. 2002;86(5):723-727. doi:10.1038/sj.bjc.6600124.

29. Kumar N, Kumari. Reproductive Factors and Risk of Breast Cancer among Patients Attending the Tertiary Care Hospital in North Bihar. Int J Sci Study. 2016;4(3):174-177.

30. Babalou A. The association of parity and breastfeeding with breast cancer: a review. Health Sci J. 2017;11(1):488. doi:10.21767/1791809X.1000488.

31. Li Cl, Beaber EF, Tang MT, Porter PL, Daling JR, Malone KE. Reproductive factors and risk of estrogen receptor positive, triple-negative, and HER2-neu overexpressing breast cancer among women 20-44 years of age. Breast Cancer Res Treat. 2013;137(2):579-587. doi:10.1007/s10549-012-2365-1.

32. Melbye M, Wohlfahrt J, Andersen AM, Westergaard T, Andersen PK. Preterm delivery and risk of breast cancer. $\mathrm{Br} J$ Cancer. 1999;80(3-4):609-613. doi:10.1038/sj.bjc.6690399.

33. Innes $K E$, Byers TE. First pregnancy characteristics and subsequent breast cancer risk among young women. Int J Cancer. 2004;112(2):306-311. doi:10.1002/ijc.20402. 